Virginia Commonwealth University vCU Scholars Compass

2011

\title{
Double exchange mediated ferromagnetic coupling between Co atoms in dicobalt complex
}

Kalpataru Pradhan

Virginia Commonwealth University, kpradhan@vcu.edu

Purusottam Jena

Virginia Commonwealth University, pjena@vcu.edu

Follow this and additional works at: http://scholarscompass.vcu.edu/phys_pubs

Part of the Physics Commons

Pradhan, K., Jena, P. Double exchange mediated ferromagnetic coupling between Co atoms in dicobalt complex. Applied Physics Letters, 99, 153105 (2011). Copyright (C) 2011 AIP Publishing LLC.

\section{Downloaded from}

http://scholarscompass.vcu.edu/phys_pubs/29

This Article is brought to you for free and open access by the Dept. of Physics at VCU Scholars Compass. It has been accepted for inclusion in Physics Publications by an authorized administrator of VCU Scholars Compass. For more information, please contact libcompass@vcu.edu. 


\title{
Double exchange mediated ferromagnetic coupling between Co atoms in dicobalt complex
}

\author{
Kalpataru Pradhan ${ }^{\text {a) }}$ and Purusottam Jena \\ Physics Department, Virginia Commonwealth University, Richmond, Virginia 23284, USA
}

(Received 19 August 2011; accepted 23 September 2011; published online 11 October 2011)

\begin{abstract}
Realization of the full technological potential of molecular magnets requires a fundamental understanding of the origin of magnetic coupling in molecular systems. Using gradient corrected density functional theory and $\mathrm{Cl}$ as a ligand we show that the magnetic coupling between Co sites in a di-cobalt complex can be manipulated. While the ferromagnetic states of $\mathrm{Co}_{2} \mathrm{Cl}_{6}$ and $\mathrm{Co}_{2} \mathrm{Cl}_{7}$ are $0.07 \mathrm{eV}$ and $0.20 \mathrm{eV}$ lower in energy than their respective antiferromagnetic states, they are nearly degenerate in $\mathrm{Co}_{2} \mathrm{Cl}_{8}$. The origin of ferromagnetic coupling is explained using the double exchange model caused by charge disproportionation. (C) 2011 American Institute of Physics. [doi:10.1063/1.3651486]
\end{abstract}

A fundamental understanding of the origin of magnetism in transition metals as well as in transition metal oxides and molecular magnets is important not only from the scientific point of view but also because of the role magnetism plays in technological applications. ${ }^{1-4}$ It is well understood that in transition metals ferromagnetic coupling is mediated by itinerant electrons. ${ }^{5,6}$ However, in transition metal oxides (TMOs) and in single-molecule magnets, electrons are localized and magnetism is often governed by the superexchange $^{7}$ mechanism that leads to antiferromagnetic coupling. However, metal atoms carrying magnetic moments in a single molecule can couple ferromagnetically through the double exchange mechanism if delocalized electrons are available. Such systems are difficult to find, and double exchange ${ }^{8}$ interaction comes into play if and only if one of the transition metal ions has an extra electron compared to the other. Thus, the only way ferromagnetic double exchange interaction can be realized in molecular magnets is by charge transfer and/or charge disproportionation between two transition metal atoms. ${ }^{9-11}$ Recent discoveries of ferromagnetic ordering in mixed-valence imidazolate-bridged di-vana$\operatorname{dium}^{12}$ and $\left[\left(\mathrm{NH}_{3}\right)_{4} \mathrm{Co}(\mathrm{OH})_{2} \mathrm{Co}\left(\mathrm{NH}_{3}\right)_{4}\right]^{4+}$ di-cobalt ${ }^{13}$ complexes show that ferromagnetic double exchange in molecular magnets can indeed be achieved via chargedisproportionation between valence-variable transition metal ions. The competition between super-exchange and doubleexchange mechanisms can give rise to exotic properties ${ }^{6,14,15}$ in TMOs. This has also been observed in metal oxides such as $\mathrm{CaCu}^{2+}{ }_{3} \mathrm{Fe}^{4+}{ }_{4} \mathrm{O}_{12}$ where the charge transfer between $\mathrm{Fe}^{3+}$ and $\mathrm{Fe}^{5+}$ ions via oxygen atom results ${ }^{16}$ a ferromagnetic double exchange coupling. This raises an important question: Can one control charge transfer/disproportionation between transition metal atoms and hence magnetism by varying the number of ligands in these molecular magnets? In this letter we demonstrate, by choosing $\mathrm{Co}_{2} \mathrm{Cl}_{n}(\mathrm{n}=6-8)$ as a model system, that magnetic coupling between two transition metal atoms can indeed be manipulated by varying the number of ligands as well as the nature of their bonding. These results, based on density functional theory, illustrate

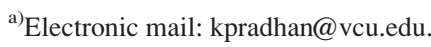

the importance of theoretical modeling to guide experiments in focused discovery of molecular magnets.

We note that Co atom with an orbital configuration of $3 \mathrm{~d}^{7} 4 \mathrm{~s}^{2}$ can exhibit oxidation states ranging from +2 to +4 . The magnetic moments associated with $\mathrm{Co}^{2+}, \mathrm{Co}^{3+}$, and $\mathrm{Co}^{4+}$ ions are, respectively, 3, 4, and $5 \mu_{\mathrm{B}}$. However, the most prevalent oxidation state of $\mathrm{Co}$ is +3 . Thus, based on the super-exchange model, one would expect $\mathrm{Co}_{2} \mathrm{Cl}_{6}$ to have antiferromagnetic ground state. We note that in the case of $\mathrm{Co}_{2} \mathrm{Cl}_{7}$ cluster, there is built-in charge disproportionation $\left(\mathrm{Co}^{3+}\right.$ and $\left.\mathrm{Co}^{4+}\right)$ and according to double exchange mechanism it should have a ferromagnetic ground state. On the contrary, $\mathrm{Co}_{2} \mathrm{Cl}_{8}$ should have an antiferromagnetic ground state if the oxidation state of $\mathrm{Co}$ in this molecule is +4 . A ferromagnetic $\mathrm{Co}_{2} \mathrm{Cl}_{6}$ cluster could exist only if there is a charge transfer/disproportionation among the Co atoms $\left(\mathrm{Co}^{3+}+\mathrm{Co}^{3+} \leftrightarrow \mathrm{Co}^{2+}+\mathrm{Co}^{4+}\right)$. To examine the extent this simple picture will hold, we have performed density functional calculations for $\mathrm{Co}_{2} \mathrm{Cl}_{\mathrm{n}}(\mathrm{n}=6-8)$ clusters.

The calculations were carried out using plane-wave pseudopotential and the supercell approach. The cluster was placed in a large cubic box of sides of $20 \AA$ in order to reduce its interaction with its images. The exchange-correlation effects were treated within the generalized gradient approximation (GGA) using the PW91 functional ${ }^{17}$ and projector augmented wave (PAW) basis set implemented in VASP code. ${ }^{18-20}$ An energy cutoff of $400 \mathrm{eV}$ was used. Brillouin zone integrations were carried out using only the Gamma point. Structures were relaxed using the conjugate gradient method for different spin multiplicities and without any symmetry constraints.

We started our calculations with $\mathrm{Co}_{2} \mathrm{Cl}_{6}$ cluster where $\mathrm{Co}$ is expected to be in +3 oxidation state and gradually increased the number of $\mathrm{Cl}$ atoms. The ground state geometries of $\mathrm{Co}_{2} \mathrm{Cl}_{6}$ cluster corresponding to ferromagnetic (FM) and antiferromagnetic (AFM) states are shown in Fig. 1 and found to have $\mathrm{D}_{2 \mathrm{~h}}$ symmetry with two bridged and four terminal $\mathrm{Cl}$ atoms. Contrary to the expectation discussed above we found that the Co atoms prefer a FM configuration which is $0.07 \mathrm{eV}$ lower in energy than the AFM state. The total spin magnetic moment of the FM state is $8 \mu_{\mathrm{B}}$. Note that a 


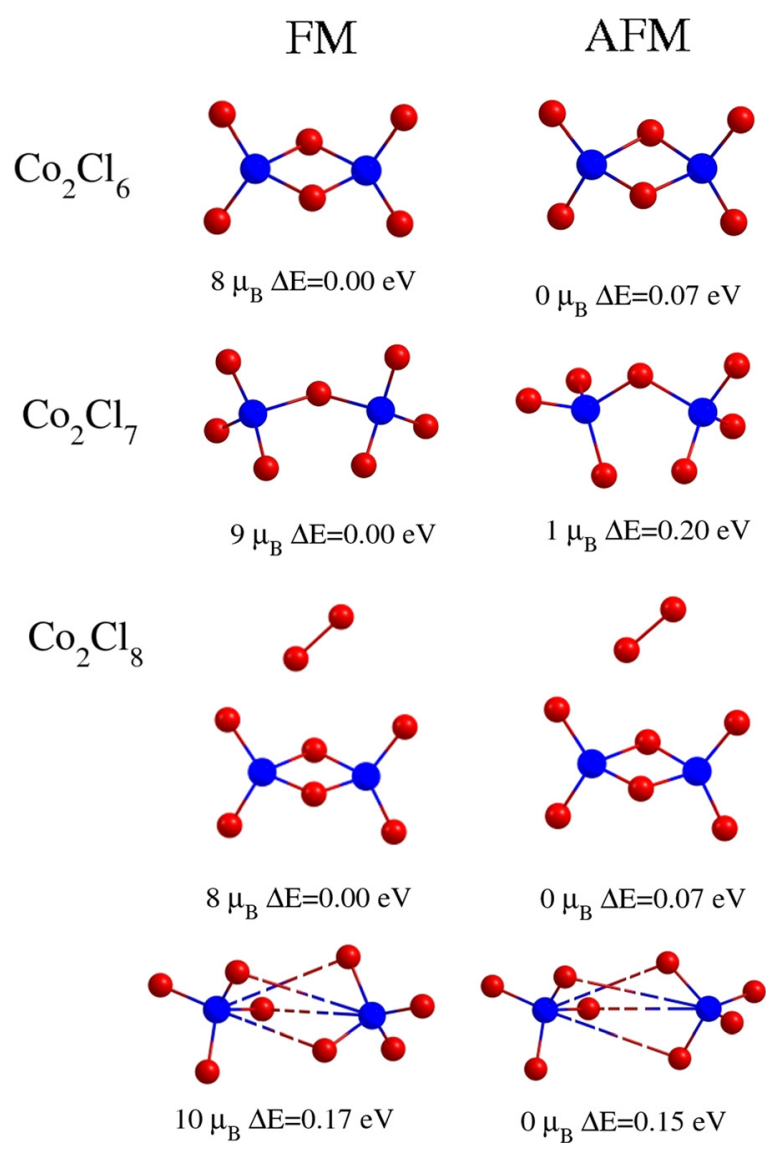

FIG. 1. (Color online) Geometries of $\mathrm{Co}_{2} \mathrm{Cl}_{\mathrm{n}}(\mathrm{n}=6-8)$ clusters corresponding to the ferromagnetic and antiferromagnetic states: $\Delta \mathrm{E}$ (refers to the relative energy of the structure measured with respect to the ground state geometry) and magnetic moments are given in $\mu_{\mathrm{B}}$. For $\mathrm{Co}_{2} \mathrm{Cl}_{8}$ cluster two isomers for each magnetic configuration are given.

magnetic moment of $4 \mu_{\mathrm{B}}$ per Co atom is consistent with the Co atom being in the +3 state which should result in the AFM ground state because of the super-exchange mechanism. However, if there is charge disproportionation, i.e., one $\mathrm{Co}$ atom would be in $\mathrm{Co}^{2+}$ state while the other in the $\mathrm{Co}^{4+}$ state, the delocalization of electrons will lead to a ferromagnetic state through the double exchange mechanism. Thus, the ultimate magnetic ordering depends upon a competition between super-exchange and double exchange mechanisms. Our computed spin magnetic moments at each of the Co atom in the FM state are $2.65 \mu_{\mathrm{B}}$. This is smaller than expected magnetic moment of $4 \mu_{\mathrm{B}}$ and is due to significant hybridization between the $\mathrm{Cl} p$ and $\mathrm{Co} d$ orbitals in the both the spin channels. This will be discussed in the following in more detail. In the AFM state the spin magnetic moments are $2.62 \mu_{\mathrm{B}}$ and $-2.62 \mu_{\mathrm{B}}$ at the Co atoms. The Co-Co bond length in the FM ground state is $3.28 \AA$ while it is $3.24 \AA$ in the AFM state.

In Fig. 2 we plot the one electron energy levels of $\mathrm{Co}_{2} \mathrm{Cl}_{6}$ cluster. The +3 valence state of each $\mathrm{Co}$ atom will result a $3 d^{6}$ atomic configuration. Ideally, one would expect $18 \mathrm{Cl} p$ and $10 \mathrm{Co} d$ orbitals in the up spin channel and $18 \mathrm{Cl}$ $p$ and 2 Co $d$ orbitals in the down spin channel. An analysis of the character of the molecular orbitals (MOs) shows the presence of prominent $p d$ hybridization in both the channels. The occupied level in spin up channel which come from $d$ orbital of Co atoms are shown in red (light) color. There are

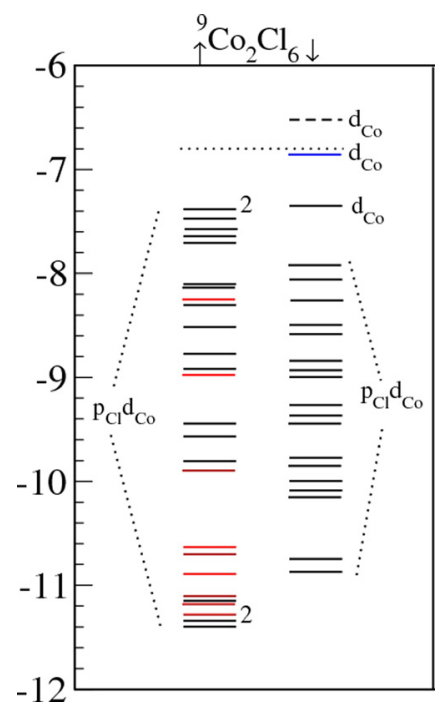

FIG. 2. (Color online) One electron energy levels (in $\mathrm{eV}$ ) in $\mathrm{Co}_{2} \mathrm{Cl}_{6}$ cluster. The solid lines represent occupied levels and the dotted lines correspond to unfilled states. The degeneracy is marked by a number next to each level. The arrows indicate the majority up and minority down spin states, and the superscript indicates the spin multiplicity.

only two occupied $d$ levels in the down spin channel without any mixing, and these are found at the top of the occupied levels. At the same time these top two occupied $d$ levels in spin down channels are separated by $0.50 \mathrm{eV}$. This indicates that the $d$ electron in the HOMO level is more itinerant than the $d$ electron present below it and can hop from one Co atom to other. This is the primary reason behind the charge disproportion and justifies the electron delocalization between $\mathrm{Co}$ atoms in the $\mathrm{Co}_{2} \mathrm{Cl}_{6}$ cluster. The electron delocalization and charge disproportionation lead to the ferromagnetic ground state as one expects from the double exchange model. In Fig. 3 we have shown a schematic diagram of our model. One of the Co atoms is in +3 state while other one is in +4 state, barring the itinerant electron. The itinerant electron can hop from one Co atom to other if the spin of the Co atoms are aligned parallel to each other. This accounts for the ferromagnetic ground state of $\mathrm{Co}_{2} \mathrm{Cl}_{6}$ cluster.

The FM ground-state and the higher energy isomer with AFM state of $\mathrm{Co}_{2} \mathrm{Cl}_{7}$ are also given in Fig. 1. The AFM state is $0.20 \mathrm{eV}$ higher in energy than the FM ground state. In both the FM and AFM geometries, each of the Co atoms are attached to three different $\mathrm{Cl}$ atoms and the 7 th $\mathrm{Cl}$ atom bridges the two Co atoms. The Co atoms prefer FM coupling. The total spin magnetic moment of $\mathrm{Co}_{2} \mathrm{Cl}_{7}$ is $9 \mu_{\mathrm{B}}$ while it is $8 \mu_{\mathrm{B}}$ in the $\mathrm{Co}_{2} \mathrm{Cl}_{6}$ cluster. In $\mathrm{Co}_{2} \mathrm{Cl}_{7}$ cluster the

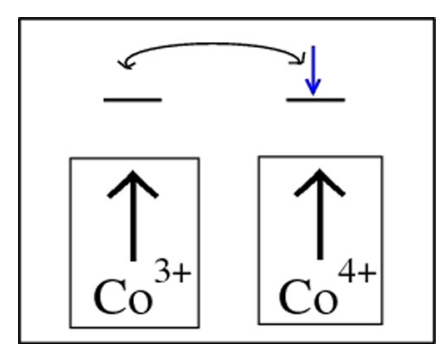

FIG. 3. (Color online) A schematic diagram of the double exchange model found in Fig. 2. 
HOMO and LUMO levels have mainly $d$ character. There is no other occupied level in the down spin channel which has significant $d$ character. The electron in the HOMO level mediates the ferromagnetic interaction between the Co atoms as we have explained in the case of $\mathrm{Co}_{2} \mathrm{Cl}_{6}$ cluster. It is important to note here that the energy difference between the ferromagnetic and antiferromagntic states in $\mathrm{Co}_{2} \mathrm{Cl}_{7}$ is three times larger than that in $\mathrm{Co}_{2} \mathrm{Cl}_{6}$ cluster.

What would happen if we add another $\mathrm{Cl}$ atom to the $\mathrm{Co}_{2} \mathrm{Cl}_{7}$ cluster? In this case if each of the Co atoms would exist in +4 oxidation state, the resulting electronic state for each Co atom will be $3 d^{5}$. The half filed $3 d$ electrons are localized and chemically more inert. Hence $\mathrm{Co}_{2} \mathrm{Cl}_{8}$ is expected to have an AFM ground state due to unavailability of any itinerant electrons. However, the ground state of $\mathrm{Co}_{2} \mathrm{Cl}_{8}$ cluster, in Fig. 1, is found to be an adduct, namely, $\mathrm{Co}_{2} \mathrm{Cl}_{6} * \mathrm{Cl}_{2}$. Consequently, the preferred oxidation state of $\mathrm{Co}$ in $\mathrm{Co}_{2} \mathrm{Cl}_{8}$ is +3 . This results in a ferromagnetic ground state and has similar electronic properties as that discussed for $\mathrm{Co}_{2} \mathrm{Cl}_{6}$ cluster.

If $\mathrm{Co}$ atom were to exist in +4 oxidation state in $\mathrm{Co}_{2} \mathrm{Cl}_{8}$, one would expect that all the $\mathrm{Cl}$ atoms bind chemically to the Co atoms. In that case one would also expect, as stated in the above, the ground state of $\mathrm{Co}_{2} \mathrm{Cl}_{8}$ to be antiferromagnetic. To examine this possibility, we searched for a higher energy isomer of $\mathrm{Co}_{2} \mathrm{Cl}_{8}$ where all the $\mathrm{Cl}$ molecules have dissociated and bound to $\mathrm{Co}$ in atomic form. These geometries are also given in Fig. 1 along with their respective energy difference from the ground state geometry. As expected, the AFM isomer is $0.02 \mathrm{eV}$ lower in energy than the FM isomer. This energy difference is only due to the antiferromagnetic interaction and is in fact very small due to the large distance between the Co atoms. But our prediction about the AFM ground state in the absence of any itinerant electron in fact holds.

In summary, calculations on $\mathrm{Co}_{2} \mathrm{Cl}_{\mathrm{n}}(\mathrm{n}=6-8)$ model clusters based on density functional theory show that the magnetic coupling in single molecule magnets can be controlled by choosing the appropriate ligands and by manipulating the way they are bound to the metal atom. We demonstrate the role charge transfer and charge disproportio- nation can play in promoting ferromagnetism in molecular magnets and how this can be achieved by attaching the right number of ligands to the transition metal atoms. These types of molecular magnets with different number of ligand atoms attached to the transition metals opens the door to design and synthesize single molecule magnets by taking advantage of the competition between the superexchange and double exchange interactions in the model systems.

This was supported in part by a grant from the Department of Energy. This research used resources of the National Energy Research Scientific Computing Center, which is supported by the Office of Science of the U.S. Department of Energy under Contract No. DE-AC02-05CH11231.

${ }^{1}$ H. B. Heersche, Z. de Groot, J. A. Folk, H. S. J. van der Zant, C. Romeike, M. R. Wegewijs, L. Zobbi, D. Barreca, E. Tondello, and A. Cornia, Phys. Rev. Lett. 96, 206801 (2006).

${ }^{2}$ D. A. Garanin and E. M. Chudnovsky, Phys. Rev. B 56, 11102 (1997).

${ }^{3}$ M. N. Leuenberger and D. Loss, Nature (London) 410, 789 (2001).

${ }^{4}$ J. J. Parks, A. R. Champagne, T. A. Costi, W. W. Shum, A. N. Pasupathy, E. Neuscamman, S. Flores-Torres, P. S. Cornaglia, A. A. Aligia, C. A. Balseiro, G. K.-L. Chan, H. D. Abruna, and D. C. Ralph, Science 328, 1370 (2010).

${ }^{5} \mathrm{~J}$. Kubler, Theory of Itinerant Electron Magnetism (Oxford University Press, New York, 2000).

${ }^{6}$ Colossal Magnetoresistive Oxides, edited by Y. Tokura (Gordon and Breach, New York, 2000).

${ }^{7}$ P. W. Anderson, Phys. Rev. 79, 350 (1950).

${ }^{8}$ C. Zener, Phys. Rev. 82, 403 (1951).

${ }^{9}$ C. Creutz and H. Taube, J. Am. Chem. Soc. 95, 1086 (1973).

${ }^{10}$ X. Q. Ding, E. L. Bominaar, E. Bill, H. Winkler, A. X. Trautwein, S. Drueke, P. Chaudhuri, and K. Wieghardt, J. Chem. Phys. 92, 178 (1990).

${ }^{11}$ V. Barone, A. Bencini, I. Ciofini, C. A. Daul, and F. Totti, J. Am. Chem. Soc. 120, 8357 (1998).

${ }^{12}$ B. Bechlars, D. M. D'Alessandro, D. M. Jenkins, A. T. Lavarone, S. D. Glover, C. P. Kubiak, and J. R. Long, Nat. Chem. 2, 362 (2010).

${ }^{13}$ Lin He and Lin Guo, Appl. Phys. Lett. 97, 182509 (2010).

${ }^{14}$ M. Imada, A. Fujimori, and Y. Tokura, Rev. Mod. Phys. 70, 1039 (1998).

${ }^{15}$ K. Pradhan, A. Mukherjee, and P. Majumdar, Phys. Rev. Lett. 99, 147206 (2007).

${ }^{16}$ I. Yamada, K. Takata, N. Hayashi, S. Shinohara, M. Azuma, S. Mori, S. Muranaka, Y. Shimakawa, and M. Takano, Angew. Chem., Int. Ed. 47, 7032 (2008).

${ }^{17}$ Y. Wang and J. P. Perdew, Phys. Rev. B 44, 13298 (1991).

${ }^{18}$ G. Kresse and J. Hafner, Phys. Rev. B 47, 558 (1993).

${ }^{19}$ P. E. Blochl, Phys. Rev. B 50, 17953 (1994).

${ }^{20}$ G. Kresse and D. Joubert, Phys. Rev. B 59, 1758 (1999). 